\title{
Identity-based deniable authenticated encryption and its application to e-mail system
}

\author{
Fagen $\mathrm{Li}^{1}$ • Zhaohui Zheng ${ }^{1}$ - Chunhua Jin ${ }^{1}$
}

Published online: 22 October 2015

(C) The Author(s) 2015. This article is published with open access at Springerlink.com

\begin{abstract}
An authenticated encryption (AE) scheme simultaneously achieves two security goals: confidentiality and authenticity. AE can be divided into symmetric $\mathrm{AE}$ and asymmetrical (public key) AE. In a symmetric AE scheme, deniability is gained automatically. However, a public key AE scheme can not gain deniability automatically; on the contrary, it provides non-repudiation. In this paper, we address a question on deniability of public key AE. Of course, we can achieve this goal by "deniable authentication followed by encryption" method. However, such method has the following two weaknesses: (1) the computational cost and communication overhead are the sum of two cryptographic primitives; (2) it is complex to design cryptographic protocols with deniable authentication and confidentiality using two cryptographic primitives. To overcome the two weaknesses, we propose a new concept called deniable authenticated encryption (DAE) that can achieve both the functions of deniable authentication and public key encryption simultaneously, at a cost significantly lower than that required by the "deniable authentication followed by encryption" method. This single cryptographic primitive can simplify the design of cryptographic protocols with deniable authentication and confidentiality. In particular, we construct an identity-based deniable authenticated encryption (IBDAE) scheme. Our construction uses tag-key encapsulation mechanism (KEM) and data encapsulation mechanism (DEM) hybrid techniques, which is more practical for true applications. We show how to construct an IBDAE scheme using an identity-based deniable authenticated tag-KEM (IBDATK)
\end{abstract}

\section{Fagen Li}

fagenli@uestc.edu.cn

1 School of Computer Science and Engineering, University of Electronic Science and Technology of China, Chengdu 611731, China and a DEM. We also propose an IBDATK scheme and prove its security in the random oracle model. For typical security level, our scheme is at least 50.7 and $22.7 \%$ faster than two straightforward "deniable authentication followed by encryption" schemes, respectively. The communication overhead is respectively reduced at least 21.3 and $31.1 \%$. An application of IBDAE to an e-mail system is described.

Keywords Security · Authenticated encryption · Identitybased cryptography $\cdot$ Deniability $\cdot$ E-mail system

\section{Introduction}

An authenticated encryption (AE) scheme simultaneously achieves two security goals: confidentiality and authenticity. $\mathrm{AE}$ can be divided into symmetric $\mathrm{AE}$ [1] and asymmetrical (public key) AE [2,3]. A symmetric AE use a keyed hash (i.e, a MAC) with some appropriate key $K_{1}$ along with a secure encryption scheme with an independent key $K_{2}$ to achieve AE. In this model, we need agree $K_{1}$ and $K_{2}$ between the sender and the receiver in advance. The authenticity of symmetric $\mathrm{AE}$ is deniable authentication since both the sender and the receiver can generate the same ciphertext. That is, the receiver can generate a ciphertext that is indistinguishable from that generated by the sender. A public key $\mathrm{AE}$ integrates public key encryption and digital signature in a single procedure to reduce the computation and communication cost. A public key AE does not automatically achieve deniable authentication since only the sender can generate a valid ciphertext. That is, a public key AE scheme provides non-repudiation. Therefore, there is an important difference about authenticity between symmetric $\mathrm{AE}$ and public key $\mathrm{AE}$. The symmetric $\mathrm{AE}$ is deniable and the public key $\mathrm{AE}$ is not deniable. 


\subsection{Motivation and contribution}

In this paper, we address a question on deniability of public key AE. Of course, we can achieve this goal by "deniable authentication followed by encryption" method. However, such method has the following two weaknesses: (1) the computational cost and communication overhead are the sum of two cryptographic primitives; (2) it is complex to design cryptographic protocols with deniable authentication and confidentiality using two cryptographic primitives. In this paper, we propose a new concept called deniable authenticated encryption (DAE) that can achieve both the functions of deniable authentication and public key encryption simultaneously, at a cost significantly lower than that required by the "deniable authentication followed by encryption" method. This single cryptographic primitive can simplify the design of cryptographic protocols with deniable authentication and confidentiality. In particular, we construct an identity-based deniable authenticated encryption (IBDAE) scheme. Our construction uses tag-key encapsulation mechanism (KEM) and data encapsulation mechanism (DEM) hybrid techniques, which is more practical for true applications. We show how to construct an IBDAE scheme using an identity-based deniable authenticated tag-KEM (IBDATK) and a DEM. We also propose an IBDATK scheme and prove its security in the random oracle model. An application of IBDAE to an e-mail system is described.

\subsection{Related work}

Here we introduce four related notions, identity-based cryptography (IBC), hybrid encryption, and deniable authentication.

IBC is introduced by Shamir [4] in 1984. Compared with the public key infrastructure (PKI), the main advantage of the IBC is the elimination of public key certificates. In the IBC, a user's public key can be derived directly from its identity information, such as telephone numbers, e-mail addresses and IP addresses. The private key of the user is generated by a trusted third party called private key generator (PKG). Authenticity of the public key is explicitly verified without requiring an attached public key certificate. The IBC is very suitable for developing a secure e-mail system. When Alice hopes to send an e-mail to Bob at bob@uestc.edu.cn, she encrypts her message using the string "bob@uestc . edu.cn". In this process, Alice does not need to obtain Bob's public key certificate. When Bob receives the encrypted e-mail, he applies for a private key from the PKG and then decrypts his e-mail. Note that unlike the PKI-based e-mail systems, Alice can send an encrypted e-mail to Bob even if Bob has not obtained his key pair information. In 2001, Boneh and Franklin [5] designed a practical identity-based encryption (IBE) scheme using bilin- ear pairings and proved its security in the random oracle model. Park and Lee [6] proposed another IBE scheme that achieves a tight security reduction to the decisional bilinear Diffie-Hellman assumption in the random oracle model. In 2002, Hess [7] constructed an identity-based signature (IBS) whose security depends on the hardness of the DiffieHellman problem in the random oracle model. In 2003, Cha and Cheon [8] designed a new IBS using gap Diffie-Hellman groups. In 2011, Hsu and Lin $[9,10]$ extended AE into the identity-based environment and constructed identity-based authenticated encryption (IBAE). However, these two IBAE schemes have non-repudiation. That is, they are not deniable. Some identity-based signcryption (IBSC) [11-13] also were proposed to achieve both confidentiality and authentication. However, these IBSC schemes still have non-repudiation.

The practical way to perform secret communication for large messages is to use hybrid encryption. The hybrid encryption splits the encryption process into two parts: one part uses public key techniques to encrypt a one-time symmetric key; the other part uses the symmetric key to encrypt the actual message. In such a construction, the public key part is called key encapsulation mechanism (KEM) and the symmetric key part is called data encapsulation mechanism (DEM). In 2003, Cramer and Shoup [14] first gave a formal analysis for the hybrid KEM-DEM construction. Then some efficient KEM-DEM constructions have been proposed [1517]. The advantage of this paradigm is that it gives a clear separation between the various parts of the cipher allowing for modular design. In [15], Abe et al. introduced tag-KEM that takes as input a tag in KEM. Note that the using of tagKEM brings simpler scheme descriptions and better generic security reductions. Bentahar et al.'s [18] extended KEM into identity-based environment and proposed several efficient constructions of identity-based KEM. In 2014, Abdalla et al. [19] discussed the relation between the notions of verifiable random functions and identity-based KEM.

Deniable authentication is different to traditional authentication and has the following two main characteristics: (1) it enables an intended receiver to identify the source of a given message; (2) the intended receiver cannot prove the source of a given message to any third party. Deniable authentication can be used in many specialized applications. For example, it can provide freedom from coercion in electronic voting systems and secure negotiation over the Internet [20,21]. In 2005, Shi and Li [22] extended deniable authentication into identity-based environment and designed an identity-based deniable authentication (IBDA) protocol. Kar [23] designed an IBDA protocol based on Diffie-Hellman problem assumption. However, Kar's protocol is interactive. An interactive protocol usually consumes more communication cost than a non-interactive protocol. In 2014, Li et al. [24] proposed a non-interactive IBDA protocol using bilinear pairings under the bilinear Diffie-Hellman assumption. In addition, their 
protocol supports batch verification that can speed up the verification of authenticators.

\subsection{Organization}

The rest of this paper is organized as follows. We define the formal model of IBDAE in Sect. 2. The IBDATK is given in Sect. 3. We show how to construct an IBDAE scheme using an IBDATK and a DEM in Sect. 4. An example of IBDATK is described in Sect. 5. We discuss the performance of our scheme in Sect. 6. A secure e-mail protocol based on IBDAE is designed in Sect. 7. Finally, the conclusions are given in Sect. 8 .

\section{IBDAE}

In this section, we give the formal definition and security notions for IBDAE.

\subsection{Syntax}

A generic IBDAE scheme consists of the following four algorithms.

- Setup is a probabilistic algorithm run by a PKG that takes as input a security parameter $k$, and outputs a master secret key $s$ and the system parameters param including a master public key $P_{\text {pub }}$. Here we assume that param are public so that we do not need to include them in other algorithms.

- Extract is a key extraction algorithm run by the PKG that takes as input an identity $I D$ and the master secret key $s$, and outputs the corresponding private key $S_{I D}$. The PKG transmits the private key to its owner in a secure way.

- Encrypt is a probabilistic deniable authenticated encryption algorithm run by a sender that takes as input a message $m$, a sender's private key $S_{I D_{s}}$ and a receiver's identity $I D_{r}$, and outputs a ciphertext $\sigma$.

- Decrypt is a deterministic deniable authenticated decryption algorithm run by a receiver that takes as input a ciphertext $\sigma$, a sender's identity $I D_{s}$ and a receiver's private key $S_{I D_{r}}$, and outputs a plaintext $m$ or the symbol $\perp$ if $\sigma$ is an invalid ciphertext between the sender and the receiver.

For consistency, we require that if $\sigma=\operatorname{Encrypt}\left(m, S_{I D_{s}}\right.$, $\left.I D_{r}\right)$, then we have $m=\operatorname{Decrypt}\left(\sigma, I D_{s}, S_{I D_{r}}\right)$. Note that here Encrypt and Decrypt are different from common Encrypt and Decrypt in public key encryption schemes. Here Encrypt means deniable authenticated encryption and Decrypt means deniable authenticated decryption. In the following contents, encryption and decryption usual mean deniable authenticated encryption and deniable authenticated decryption, respectively.

\subsection{Security notions}

An IBDAE scheme should satisfy confidentiality and deniable authentication. Here we give two games to capture the two security properties.

The standard adopted security notion for confidentiality is the indistinguishability against adaptive chosen ciphertext attack (IND-CCA2). We apply this notion to our IBDAE. We consider the following game (Game-I) played between a challenger $\mathcal{C}$ and an adversary $\mathcal{A}$.

- Initial $\mathcal{C}$ runs Setup algorithm with a security parameter $k$ and sends the system parameters param to $\mathcal{A}$.

- Phase $1 \mathcal{A}$ performs a polynomially bounded number of queries (these queries may be made adaptively, i.e. each query may depend on the answer to the previous queries).

- Key extraction queries $\mathcal{A}$ chooses an identity I D. $\mathcal{C}$ runs Extract algorithm and sends the corresponding private key $S_{I D}$ to $\mathcal{A}$.

- Encryption queries $\mathcal{A}$ produces a plaintext $m$ and two identities $I D_{i}$ and $I D_{j} \cdot \mathcal{C}$ first runs Extract algorithm to generate the sender's private key $S_{I D_{i}}$. Then $\mathcal{C}$ runs Encrypt $\left(m, S_{I D_{i}}, I D_{j}\right)$ algorithm and sends $\sigma$ to $\mathcal{A}$.

- Decryption queries $\mathcal{A}$ produces a ciphertext $\sigma$ and two identities $I D_{i}$ and $I D_{j} \cdot \mathcal{C}$ first runs Extract algorithm to generate the receiver's private key $S_{I D_{j}}$. Then $\mathcal{C}$ runs Decrypt $\left(\sigma, I D_{i}, S_{I D_{j}}\right)$ algorithm and sends the result to $\mathcal{A}$ (this result can be the $\perp$ symbol if $\sigma$ is an invalid ciphertext).

- Challenge $\mathcal{A}$ decides when phase 1 ends. $\mathcal{A}$ generates two equal length plaintexts $m_{0}$ and $m_{1}$ and two identities $I D_{s}$ and $I D_{r}$ on which it wants to be challenged. It can not have asked the private key corresponding to $I D_{r}$ in the phase $1 . \mathcal{C}$ takes a random bit $\beta \in\{0,1\}$ and computes $\sigma^{*}=\operatorname{Encrypt}\left(m_{\beta}, S_{I D_{s}}, I D_{r}\right)$ which is sent to $\mathcal{A}$.

- Phase $2 \mathcal{A}$ can ask a polynomially bounded number of queries adaptively again as in the phase 1 . This time, it can not ask a key extraction query on $I D_{r}$ and can not ask a decryption query on $\left(\sigma^{*}, I D_{s}, I D_{r}\right)$ to obtain the corresponding plaintext.

- Guess $\mathcal{A}$ produces a bit $\beta^{\prime}$ and wins the game if $\beta^{\prime}=\beta$.

The advantage of $\mathcal{A}$ is defined as $\operatorname{Adv}(\mathcal{A})=\mid 2 \operatorname{Pr}\left[\beta^{\prime}=\beta\right]-$ 1 , where $\operatorname{Pr}\left[\beta^{\prime}=\beta\right]$ denotes the probability that $\beta^{\prime}=\beta$. 
Definition 1 An adversary $\mathcal{A}$ is said to be an $\left(\epsilon_{d a e}, t, q_{k}\right.$, $q_{e}, q_{d}$ )-attacker of an IBDAE scheme if $\mathcal{A}$ has advantage at least $\epsilon_{d a e}$ in the Game-I, runs in time at most $t$, and makes at most $q_{k}$ key extraction queries, $q_{e}$ encryption queries and $q_{d}$ decryption queries. An IBDAE scheme is said to be $\left(\epsilon_{d a e}, t, q_{k}, q_{e}, q_{d}\right)$-IND-CCA2 secure if no $\left(\epsilon_{d a e}, t, q_{k}, q_{e}, q_{d}\right)$-attacker exists.

Note that $\mathcal{A}$ is allowed to make a key extraction query on identity $I D_{s}$ in the Game-I. It ensures the forward security of the scheme, i.e. confidentiality is preserved in case the sender's private key becomes compromised.

For the deniable authentication, we adopt deniable authentication against adaptive chosen messages attack (DA-CMA) notion in [24]. We consider the following game (Game-II) played between a challenger $\mathcal{C}$ and an adversary $\mathcal{F}$.

- Initial $\mathcal{C}$ runs Setup algorithm with a security parameter $k$ and sends the system parameters param to $\mathcal{F}$.

- Attack $\mathcal{F}$ performs a polynomially bounded number of queries just like in the Game-I.

- Forgery $\mathcal{F}$ produces a triple $\left(\sigma^{*}, I D_{S}, I D_{r}\right)$ and wins the game if the following conditions hold:

1. $\operatorname{Decrypt}\left(\sigma^{*}, I D_{s}, S_{I D_{r}}\right)=m^{*}$.

2. $\mathcal{F}$ has not asked key extraction queries on $I D_{s}$ and $I D_{r}$.

3. $\mathcal{F}$ has not asked an encryption query on $\left(m^{*}, I D_{s}\right.$, $\left.I D_{r}^{\prime}\right)$. Here $I D_{r}^{\prime}$ may be different from $I D_{r}$.

The advantage of $\mathcal{F}$ is defined as the probability that it wins.

Definition 2 An adversary $\mathcal{F}$ is said to be an $\left(\epsilon_{d a e}, t, q_{k}\right.$, $q_{e}, q_{d}$ )-forger of an IBDAE scheme if $\mathcal{F}$ has advantage at least $\epsilon_{d a e}$ in the Game-II, runs in time at most $t$, and makes at most $q_{k}$ key extraction queries, $q_{e}$ encryption queries and $q_{d}$ decryption queries. An IBDAE scheme is said to be $\left(\epsilon_{d a e}, t, q_{k}, q_{e}, q_{d}\right)$-DA-CMA secure if no $\left(\epsilon_{d a e}, t, q_{k}, q_{e}, q_{d}\right)$-forger exists.

Note that $\mathcal{F}$ is not allowed to make a key extraction query on identity $I D_{r}$ in the Game-II. This condition is necessary to obtain the deniability property. The sender can deny its action since the receiver also can generate a valid ciphertext. This is the main difference between deniable authentication in DAE and undeniable authentication in digital signature.

\section{IBDATK}

In this section, we give the formal definition and security notions for identity-based deniable authenticated tag-KEM (IBDATK). IBDATK can be considered as an identity-based tag-KEM with deniable authentication property. IBDATK should provide two important security properties: confidentiality and deniable authentication.

\subsection{Syntax}

A generic IBDATK scheme consists of the following five algorithms.

- Setup is a probabilistic algorithm run by a PKG that takes as input a security parameter $k$, and outputs the system's parameters param, including a master public key $P_{p u b}$ and a master secret key $s$. We also assume that param are public so that we do not need to include them in other algorithms.

- Extract is a key extraction algorithm run by the PKG that takes as input an identity $I D$ and the master secret key $s$, and outputs the corresponding private key $S_{I D}$. The PKG transmits the private key to its owner in a secure way.

- Sym is a probabilistic symmetric key generation algorithm run by a sender that takes as input a sender's private key $S_{I D_{s}}$ and a receiver's identity $I D_{r}$, and outputs a symmetric key $K$ together with internal state information $\omega$. Here $K \in \mathcal{K}_{\text {IBDATK }}$ is a key in the space of possible session keys at a given security level. $\mathcal{K}_{\text {IBDATK }}$ is the key space.

- Encap is a probabilistic key encapsulation algorithm which takes as input the state information $\omega$ and an arbitrary tag $\tau$, and returns an encapsulation $\psi \in \mathcal{E}_{\text {IBDATK }}$. Here $\mathcal{E}_{\text {IBDATK }}$ is the encapsulation space.

- Decap is a deterministic decapsulation algorithm run by a receiver that takes as input an encapsulation $\psi$, a tag $\tau$, a sender's identity $I D_{S}$ and a receiver's private key $S_{I D_{r}}$, and outputs the corresponding key $K$ or a special symbol $\perp$ indicating invalid encapsulation.

We make the consistency constraint that if $(K, \omega)=$ $\operatorname{Sym}\left(S_{I D_{s}}, I D_{r}\right)$ and $\psi=\operatorname{Encap}(\omega, \tau)$, then $K=$ $\operatorname{Decap}\left(\psi, \tau, I D_{s}, S_{I D_{r}}\right)$.

\subsection{Security notions}

An IBDATK scheme should satisfy confidentiality and deniable authentication. We give the formal security definition here. For the confidentiality, we consider the following game (Game-III) played between a challenger $\mathcal{C}$ and an adversary $\mathcal{A}$.

- Initial $\mathcal{C}$ runs Setup algorithm with a security parameter $k$ and sends the system parameters param to $\mathcal{A}$.

- Phase $1 \mathcal{A}$ performs a polynomially bounded number of queries (these queries may be made adaptively, i.e. 
each query may depend on the answer to the previous queries).

- Key extraction queries $\mathcal{A}$ chooses an identity ID.C runs Extract algorithm and sends the corresponding private key $S_{I D}$ to $\mathcal{A}$.

- Symmetric key generation queries $\mathcal{A}$ produces a sender's identity $I D_{i}$ and a receiver's identity $I D_{j}$. $\mathcal{C}$ first runs Extract algorithm to generate the sender's private key $S_{I D_{i}}$ and runs $(K, \omega)=$ $\operatorname{Sym}\left(S_{I D_{i}}, I D_{j}\right)$. It then stores the value $\omega$ (hidden from the view of the adversary, and overwriting any previously stored values), and sends the symmetric key $K$ to $\mathcal{A}$.

- Key encapsulation queries $\mathcal{A}$ produces an arbitrary $\operatorname{tag} \tau$. $\mathcal{C}$ checks whether there exists a stored value $\omega$. If not, it returns $\perp$ and terminates. Otherwise it erases the value from storage and returns $\psi=\operatorname{Encap}(\omega, \tau)$ to $\mathcal{A}$.

- Key decapsulation queries $\mathcal{A}$ chooses an encapsulation $\psi$, a tag $\tau$, a sender's identity $I D_{i}$ and a receiver's identity $I D_{j} . \mathcal{C}$ first runs Extract algorithm to generate the receiver's private key $S_{I D_{j}}$. Then $\mathcal{C}$ runs $\operatorname{Decap}\left(\psi, \tau, I D_{i}, S_{I D_{j}}\right)$ algorithm and sends the result to $\mathcal{A}$.

- Challenge $\mathcal{A}$ decides when phase 1 ends. $\mathcal{A}$ generates a sender's identity $I D_{s}$ and a receiver's identity $I D_{r}$ on which it wishes to be challenged. The identity $I D_{r}$ should not appear in any key extraction queries in phase 1. $\mathcal{C}$ first runs $\left(K_{1}, \omega^{*}\right)=\operatorname{Sym}\left(S_{I D_{s}}, I D_{r}\right)$. Then $\mathcal{C}$ chooses $K_{0} \in \mathcal{K}_{\text {IBDATK }}$ and a bit $\beta \in\{0,1\}$ randomly, and sends $K_{\beta}$ to $\mathcal{A}$. When $\mathcal{A}$ receives $K_{\beta}$, it may ask the same queries as previously. Then $\mathcal{A}$ generates a tag $\tau^{*}$. Finally, $\mathcal{C}$ computes $\psi^{*}=\operatorname{Encap}\left(\omega^{*}, \tau^{*}\right)$ and sends it to $\mathcal{A}$ as a challenge encapsulation.

- Phase $2 \mathcal{A}$ can ask a polynomially bounded number of queries adaptively again as in phase 1 with the restriction that it can not ask a key extraction query on $I D_{r}$ and can not ask a decapsulation query on $\left(\psi^{*}, \tau^{*}, I D_{s}, I D_{r}\right)$ to obtain the corresponding key.

- Guess $\mathcal{A}$ produces a bit $\beta^{\prime}$ and wins the game if $\beta^{\prime}=\beta$.

The advantage of $\mathcal{A}$ is defined $\operatorname{adv}(\mathcal{A})=\mid 2 \operatorname{Pr}\left[\beta^{\prime}=\beta\right]-$ $1 \mid$, where $\operatorname{Pr}\left[\beta^{\prime}=\beta\right]$ denotes the probability that $\beta^{\prime}=\beta$.

Definition 3 An adversary $\mathcal{A}$ is said to be an $\left(\epsilon_{\text {datk }}, t, q_{k}, q_{s}\right.$, $q_{e}, q_{d}$ )-attacker of an IBDATK scheme if $\mathcal{A}$ has advantage at least $\epsilon_{\text {datk }}$ in the Game-III, runs in time at most $t$, and asks at most $q_{k}$ key extraction queries, $q_{s}$ symmetric key generation queries, $q_{e}$ key encapsulation queries and $q_{d}$ key decapsulation queries. An IBDATK scheme is said to be $\left(\epsilon_{d a t k}, t, q_{k}, q_{s}, q_{e}, q_{d}\right)$-IND-CCA2 secure if no $\left(\epsilon_{\text {datk }}, t, q_{k}, q_{s}, q_{e}, q_{d}\right)$-attacker exists.
For deniable authentication, we consider the following game (Game-IV) played between a challenger $\mathcal{C}$ and an adversary $\mathcal{F}$.

- Initial $\mathcal{C}$ runs Setup algorithm with a security parameter $k$ and sends the system parameters param to $\mathcal{F}$.

- Attack $\mathcal{F}$ performs a polynomially bounded number of queries just like in the Game-III.

- Forgery $\mathcal{F}$ produces a quaternion $\left(\psi^{*}, \tau^{*}, I D_{s}, I D_{r}\right)$ and wins the game if the following conditions hold:

1. $\operatorname{Decap}\left(\psi^{*}, \tau^{*}, I D_{s}, S_{I D_{r}}\right)=K^{*}$.

2. $\mathcal{F}$ has not asked key extraction queries on $I D_{s}$ and $I D_{r}$.

3. $\mathcal{F}$ has not asked a key encapsulation query on $\tau^{*}$.

The advantage of $\mathcal{F}$ is defined as the probability that it wins.

Definition 4 An adversary $\mathcal{F}$ is said to be an $\left(\epsilon_{\text {datk }}, t, q_{k}, q_{s}\right.$, $q_{e}, q_{d}$ )-forger of an IBDATK scheme if $\mathcal{F}$ has advantage at least $\epsilon_{\text {datk }}$ in the Game-IV, runs in time at most $t$, and asks at most $q_{k}$ key extraction queries, $q_{s}$ symmetric key generation queries, $q_{e}$ key encapsulation queries and $q_{d}$ key decapsulation queries. An IBDATK scheme is said to be $\left(\epsilon_{\text {datk }}, t, q_{k}, q_{s}, q_{e}, q_{d}\right)$-DA-CMA secure if no $\left(\epsilon_{\text {datk }}, t, q_{k}, q_{s}, q_{e}, q_{d}\right)$-forger exists.

\section{A hybrid IBDAE scheme}

We can combine an IBDATK scheme IBDATK with a DEM scheme DEM (the definition of DEM can be found in Appendix 1) to form a hybrid IBDAE scheme IBDAE. Our method is described in Fig. 1. Note that the tag is the ciphertext output by the DEM. Such construction yields simpler scheme descriptions and better generic security reductions.

We give the security results for such construction in Theorems 1 and 2 .

Theorem 1 Let IBDAE be a hybrid IBDAE scheme constructed from an IBDATK scheme IBDATK and a DEM scheme DEM. If the IBDATK is $\left(\epsilon_{\text {datk }}, t, q_{k}, q_{s}, q_{e}, q_{d}\right)$-IND$C C A 2$ secure and the DEM is $\left(\epsilon_{\text {dem }}, \bar{t}\right)$-IND-PA secure, then the IBDAE is $\left(\epsilon_{d a e}, t^{\prime}, q_{k}^{\prime}, q_{e}^{\prime}, q_{d}^{\prime}\right)$-IND-CCA2 secure, where $\epsilon_{\text {datk }} \geq\left(\epsilon_{\text {dae }}-\epsilon_{\text {dem }}\right) / 2, t=t^{\prime}+\bar{t}+O\left(q_{e}^{\prime} T_{\text {enc }}+q_{d}^{\prime} T_{d e c}\right)$, $q_{k}=q_{k}^{\prime}, q_{s}=q_{e}=q_{e}^{\prime}, q_{d}=q_{d}^{\prime}$. Here $T_{\text {enc }}$ and $T_{\text {dec }}$ are the maximum time for computing an encryption in DEM and a decryption in DEM.

Proof See Appendix 2.

Theorem 2 Let IBDAE be a hybrid IBDAE scheme constructed from an IBDATK scheme IBDATK and a DEM scheme DEM. If the IBDATK is $\left(\epsilon_{\text {datk }}, t, q_{k}, q_{s}, q_{e}, q_{d}\right)$-DACMA secure, then the IBDAE is $\left(\epsilon_{d a e}, t^{\prime}, q_{k}^{\prime}, q_{e}^{\prime}, q_{d}^{\prime}\right)-D A$ $C M A$ secure, where $\epsilon_{\text {datk }} \geq \epsilon_{\text {dae }}, t=t^{\prime}+O\left(q_{e}^{\prime} T_{\text {enc }}+\right.$ 
Fig. 1 Construction of IBDAE from IBDATK and DEM
IBDAE.Setup: On input a security parameter $k$ :

1. $($ param,$s)=$ IBDATK. $\operatorname{Setup}(k)$

2. Output the system parameters param and the master secret key $s$

IBDAE.Extract: On input an identity $I D \in\{0,1\}^{*}$ and the master secret key $s$ :

1. $S_{I D}=$ IBDATK.Extract $(I D, s)$

2. Output the private key $S_{I D}$ for the identity $I D$

IBDAE. Encrypt: On input a message $m \in\{0,1\}^{*}$, a sender's private key $S_{I D_{s}}$ and a receiver's identity $I D_{r}$ :

1. $(K, \omega)=$ IBDATK.Sym $\left(S_{I D_{s}}, I D_{r}\right)$

2. $c=\operatorname{DEM} \cdot \operatorname{Enc}(K, m)$

3. $\psi=\operatorname{IBDATK} \operatorname{Encap}(\omega, c)$

4. Output the ciphertext $\sigma=(\psi, c)$

IBDAE. Decrypt: On input a ciphertext $\sigma$, a sender's identity $I D_{s}$ and a receiver private key $S_{I D_{r}}$ :

1. $K=$ IBDATK.Decap $\left(\psi, c, I D_{s}, S_{I D_{r}}\right)$

2. If $K=\perp$, then output $\perp$ and stop

3. $m=\operatorname{DEM} \cdot \operatorname{Dec}(K, c)$

4. Output the message $m$ $\left.q_{d}^{\prime} T_{\text {dec }}\right), q_{k}=q_{k}^{\prime}, q_{s}=q_{e}=q_{e}^{\prime}, q_{d}=q_{d}^{\prime}$. Here $T_{\text {enc }}$ and $T_{d e c}$ are the maximum time for computing an encryption in $\mathrm{DEM}$ and a decryption in DEM.

Proof See Appendix 3.

\section{An efficient IBDATK scheme}

In this section, we propose an efficient IBDATK scheme using bilinear pairings. We first describe the basic definition and properties of the bilinear pairings. The we give the IBDATK scheme and discuss its consistency, deniability and security.

\subsection{Bilinear pairings}

Let $G_{1}$ be a cyclic additive group generated by $P$, whose order is a prime $q$, and $G_{2}$ be a cyclic multiplicative group of the same order $q$. A bilinear pairing is a map $\hat{e}: G_{1} \times G_{1} \rightarrow$ $G_{2}$ with the following properties:

1. Bilinearity $\hat{e}(a P, b Q)=\hat{e}(P, Q)^{a b}$ for all $P, Q \in G_{1}$, $a, b \in \mathbb{Z}_{q}^{*}$.

2. Non-degeneracy There are $P$ and $Q \in G_{1}$ such that $\hat{e}(P, Q) \neq 1$, where 1 is the identity element of group $G_{2}$.

3. Computability There is an efficient algorithm to compute $\hat{e}(P, Q)$ for all $P, Q \in G_{1}$.

The modified Weil pairing and the Tate pairing [5,7,8] are admissible maps of this kind. The security of our scheme described here relies on the hardness of the following problems.

Given two groups $G_{1}$ and $G_{2}$ of the same prime order $q$, a bilinear map $\hat{e}: G_{1} \times G_{1} \rightarrow G_{2}$ and a generator $P$ of $G_{1}$, the decisional bilinear Diffie-Hellman (DBDH) problem in $\left(G_{1}, G_{2}, \hat{e}\right)$ is to decide whether $\theta=\hat{e}(P, P)^{a b c}$ or not given $(P, a P, b P, c P)$ and an element $\theta \in G_{2}$. We define the advantage of an adversary $\mathcal{C}$ against the DBDH like this

$$
\begin{aligned}
\operatorname{Adv}(\mathcal{C})= & \mid P_{a, b, c, \in \mathbb{Z}_{q}^{*}, \theta \in G_{2}}[\mathcal{C}(P, a P, b P, c P, \theta)=1] \\
& -P_{a, b, c, \in \mathbb{Z}_{q}^{*}}\left[\mathcal{C}\left(P, a P, b P, c P, \hat{e}(P, P)^{a b c}\right)=1\right] \mid .
\end{aligned}
$$

Definition 5 The $\left(\epsilon_{d b d h}, t\right)-\mathrm{DBDH}$ assumption holds if no $t$-polynomial time adversary $\mathcal{C}$ has advantage at least $\epsilon_{d b d h}$ in solving the DBDH problem.

Given two groups $G_{1}$ and $G_{2}$ of the same prime order $q$, a bilinear map $\hat{e}: G_{1} \times G_{1} \rightarrow G_{2}$ and a generator $P$ of $G_{1}$, the bilinear Diffie-Hellman (BDH) problem in $\left(G_{1}, G_{2}, \hat{e}\right)$ is to compute $h=\hat{e}(P, P)^{a b c}$ given $(P, a P, b P, c P)$.

Definition 6 The $\left(\epsilon_{b d h}, t\right)-\mathrm{BDH}$ assumption holds if no $t$ polynomial time adversary $\mathcal{C}$ has advantage at least $\epsilon_{b d h}$ in solving the $\mathrm{BDH}$ problem.

\subsection{Our scheme}

Our scheme consists of the following five algorithms.

- Setup Define $G_{1}, G_{2}$ and $\hat{e}$ as in Sect. 5.1. Let $H_{1}, H_{2}$ and $H_{3}$ be three hash functions where $H_{1}:\{0,1\}^{*} \rightarrow$ 
$G_{1}, H_{2}: G_{2} \rightarrow\{0,1\}^{n}$ and $H_{3}:\{0,1\}^{*} \times G_{2} \rightarrow \mathbb{Z}_{q}^{*}$. Here $n$ is the key length of a DEM and $q \geq 2^{k}$, where $k$ is a security parameter. Let $P$ be a generator of $G_{1}$. The PKG chooses a master secret key $s \in \mathbb{Z}_{q}^{*}$ randomly and computes $P_{\text {pub }}=s P$. The PKG publishes system parameters param $=\left\{G_{1}, G_{2}, q, n, \hat{e}, P, P_{p u b}, H_{1}, H_{2}, H_{3}\right\}$ and keeps the master secret key $s$ secret.

- Extract Given an identity $I D$, the PKG computes the corresponding private key $S_{I D}=s Q_{I D}$ and sends it to its owner in a secure way. Here $Q_{I D}=H_{1}(I D)$.

- Sym Given a sender's private key $S_{I D_{s}}$ and a receiver's identity $I D_{r}$, this algorithm works as follows.

1. Choose $x$ from $\mathbb{Z}_{q}^{*}$ randomly.

2. Compute $z=\hat{e}\left(P_{p u b}, Q_{I D_{r}}\right)^{x}$.

3. Output $K=H_{2}(z)$ and $\omega=\left(x, z, S_{I D_{s}}, I D_{s}, I D_{r}\right)$.

- Encap Given the state information $\omega$ and an arbitrary $\operatorname{tag} \tau$, this algorithm works as follows.

1. Compute $u=H_{3}(\tau, z)$.

2. Compute $V=u S_{I D_{s}}+x P_{p u b}$ and $T=\hat{e}\left(V, Q_{I D_{r}}\right)$.

3. Compute $R=u Q_{I D_{s}}$.

4. Output $\psi=(R, T)$.

- Decap Given an encapsulation $\psi=(R, T)$, a tag $\tau$, a sender's identity $I D_{S}$ and a receiver's private key $S_{I D_{r}}$, this algorithm works as follows.

1. Compute $z=T / \hat{e}\left(R, S_{I D_{r}}\right)$.

2. Compute $u=H_{3}(\tau, z)$.

3. If $R=u Q_{I D_{s}}$, output the $K=H_{2}(z)$, otherwise output the symbol $\perp$.

\subsection{Consistency}

The consistency of our scheme can be easily verified by the following equations.

$$
\begin{aligned}
z & =\frac{T}{\hat{e}\left(R, S_{I D_{r}}\right)}=\frac{\hat{e}\left(V, Q_{I D_{r}}\right)}{\hat{e}\left(R, S_{I D_{r}}\right)}=\frac{\hat{e}\left(u S_{I D_{s}}+x P_{p u b}, Q_{I D_{r}}\right)}{\hat{e}\left(R, S_{I D_{r}}\right)} \\
& =\frac{\hat{e}\left(u S_{I D_{s}}, Q_{I D_{r}}\right) \hat{e}\left(x P_{p u b}, Q_{I D_{r}}\right)}{\hat{e}\left(R, S_{I D_{r}}\right)} \\
& =\frac{\hat{e}\left(u Q_{I D_{s}}, S_{I D_{r}}\right) \hat{e}\left(P_{p u b}, Q_{I D_{r}}\right)^{x}}{\hat{e}\left(R, S_{I D_{r}}\right)} \\
& =\left(P_{p u b}, Q_{I D_{r}}\right)^{x} .
\end{aligned}
$$

\subsection{Deniability}

The receiver with private key $S_{I D_{r}}$ can generate an encapsulation that is indistinguishable from that generated by the sender with private key $S_{I D_{s}}$. To simulate the transcripts on a tag $\tau$, the receiver performs the steps below.
1. Choose $\bar{x}$ from $\mathbb{Z}_{q}^{*}$ randomly.

2. Compute $\bar{z}=\hat{e}\left(P_{p u b}, Q_{I D_{r}}\right)^{\bar{x}}$.

3. Compute $\bar{u}=H_{3}(\tau, \bar{z})$.

4. Compute $\bar{R}=\bar{u} Q_{I D_{s}}$.

5. Compute $\bar{T}=\bar{z} \cdot \hat{e}\left(\bar{R}, S_{I D_{r}}\right)$.

$\bar{\psi}=(\bar{R}, \bar{T})$ generated by the receiver is indistinguishable from $\psi=(R, T)$ that is generated by the sender according to the Encap algorithm in Sect. 5.2. Let $\psi^{\prime}=\left(R^{\prime}, T^{\prime}\right)$ be an encapsulation that is randomly chosen in the set of all valid sender's encapsulation intended to receiver. The probability $\operatorname{Pr}\left[\bar{\psi}=\psi^{\prime}\right]$ is $1 /(q-1)$ because $\bar{\psi}$ is generated from a randomly chosen value $\bar{x} \in \mathbb{Z}_{q}^{*}$. Likewise, the probability $\operatorname{Pr}\left[\psi=\psi^{\prime}\right]$ has the same value $1 /(q-1)$ because it is generated from $x \in \mathbb{Z}_{q}^{*}$. That is, both distributions are the same.

\subsection{Security}

Theorem 3 In the random oracle model, if an adversary $\mathcal{A}$ has a non-negligible advantage $\epsilon_{\text {datk }}$ against the INDCCA2 security of the proposed scheme when running in a time $t$ and performing $q_{k}$ key extraction queries, $q_{s}$ symmetric key generation queries, $q_{e}$ key encapsulation queries, $q_{d}$ key decapsulation queries and $q_{H_{i}}$ queries to oracles $H_{i}(i=$ $1,2,3)$, then there exists an algorithm $\mathcal{C}$ that can solve the $D B D H$ problem with an advantage

$\epsilon_{d b d h} \geq \frac{\epsilon-q_{d} / 2^{k-1}}{2 q_{H_{1}}}$

in a time $t^{\prime} \leq t+O\left(q_{s}+q_{e}+q_{d}\right) t_{p}$, where $t_{p}$ denotes the cost for one pairing operation.

Proof See Appendix 4.

Theorem 4 In the random oracle model, if an adversary $\mathcal{F}$ is able to win the Game-IV with an advantage $\epsilon \geq$ $5\left(q_{e}+1\right)\left(q_{e}+q_{H_{3}}\right) q_{H_{1}} /\left(2^{k}-1\right)$ within a time $t$ for $a$ security parameter $k$ and asking at most $q_{k}$ key extraction queries, $q_{s}$ symmetric key generation queries, $q_{e}$ key encapsulation queries, $q_{d}$ key decapsulation queries and $q_{H_{i}}$ queries to oracles $H_{i}(i=1,2,3)$, then there exists an algorithm $\mathcal{C}^{\prime}$ that can solve the $\mathrm{BDH}$ problem in expected time $t \leq 60343 q_{H_{3}} q_{H_{1}} 2^{k} t / \epsilon\left(2^{k}-1\right)$.

Proof See Appendix 5.

\section{Performance discussion}

We compare major computational cost, communication overhead, and formal security of straightforward "deniable authentication followed by encryption" method with those 
Table 1 Performance comparison

\begin{tabular}{|c|c|c|c|c|c|}
\hline \multirow[t]{2}{*}{ Schemes } & \multicolumn{3}{|c|}{ Computational cost } & \multirow[t]{2}{*}{ Communication overhead } & \multirow[t]{2}{*}{ Formal security } \\
\hline & PM & $\mathrm{EC}$ & $\mathrm{PC}$ & & \\
\hline $\mathrm{SL}+\mathrm{BF}$ & 6 & 3 & 7 & $3\left|G_{1}\right|+\left|\mathbb{Z}_{q}^{*}\right|+|M A C|+2|m|$ & No \\
\hline $\mathrm{LXJ}+\mathrm{BF}$ & 5 & 1 & 4 & $2\left|G_{1}\right|+\left|G_{2}\right|+2|m|$ & No \\
\hline Ours & 4 & 1 & 3 & $\left|G_{1}\right|+\left|G_{2}\right|+|m|$ & Yes \\
\hline
\end{tabular}

of our scheme in Table 1. For convenience, we use SL+BF to denote the straightforward method based on the deniable authentication in [22] and the encryption scheme in [5] and $\mathrm{LXJ}+\mathrm{BF}$ to denote the straightforward method based on the deniable authentication in [24] and the encryption scheme in [5]. We denote by PM the point multiplication in $G_{1}, \mathrm{EC}$ the exponentiation computation in $G_{2}$ and PC the pairing computation. The other operations are ignored in Table 1 since these operations take the most running time of the whole algorithm. $|x|$ denotes the number of bits of $x$. From Table 1, we find that our scheme has the least computational cost and communication overhead. In addition, our scheme has the formal security proof and the straightforward method has no such property.

We give a quantitative analysis for the computational cost and communication overhead. We use PBC Type A pairing [25] in this analysis. The Type A pairing is constructed on the curve

$y^{2} \equiv\left(x^{3}+x\right) \bmod p$

for some prime $p \equiv 3 \bmod 4$, where the embedding degree is 2 and the order of $G_{1}$ is $q$. In this analysis, we use three kinds of parameters that represents 80-bit, 112-bit and 128bit AES [26] key size security level, respectively. Table 2 gives the specification for different security level of this analysis.

Figure 2 gives the implementation time of $\mathrm{SL}+\mathrm{BF}$, LXJ+BF and our scheme on ThinkPad T430s that is equipped with an Intel Core i5 $3210 \mathrm{M} 2.5 \mathrm{GHz}$ machine with $4 \mathrm{G}$ RAM. From Fig. 2, we know that our scheme is $\frac{169.8-83.7}{169.8}=$ $50.7 \%$ faster and $\frac{108.3-83.7}{108.3}=22.7 \%$ faster than SL+BF and $\mathrm{LXJ}+\mathrm{BF}$, respectively, at 80-bit security level. At 112bit security level, our scheme is $\frac{835.5-407.2}{835.5}=51.2 \%$ faster and $\frac{528.2-407.2}{528.2}=22.9 \%$ faster than $\mathrm{SL}+\mathrm{BF}$ and

Table 2 Specification for different security level of this analysis (bits)

\begin{tabular}{lcl}
\hline Security level & Size of $p$ & Size of $q$ \\
\hline 80-bit & 512 & 160 \\
112-bit & 1024 & 224 \\
128-bit & 1536 & 256 \\
\hline
\end{tabular}

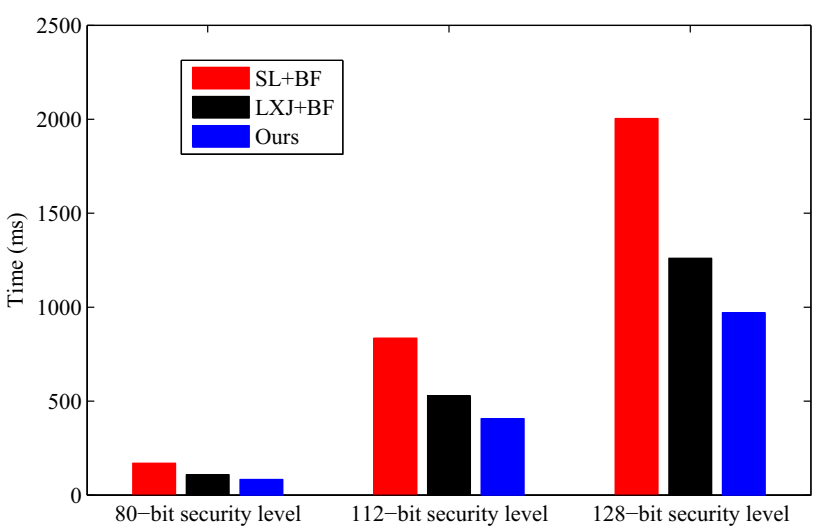

Fig. 2 The implementation time

LXJ+BF, respectively. At 128-bit security level, our scheme is $\frac{2003.9-970.3}{2003.9}=51.5 \%$ faster and $\frac{1260.6-970.3}{1260.6}=23.0 \%$ faster than $\mathrm{SL}+\mathrm{BF}$ and $\mathrm{LXJ}+\mathrm{BF}$, respectively.

Now we consider the communication overhead. Note that for 80-bit, 112-bit and 128-bit security level, the corresponding output sizes of MAC are 160 bits, 224 bits and 256 bits, respectively. When we adopt the 80-bit security level, the size of $p$ is 512 bits. So the size of an element in group $G_{1}$ is 1024 bits using an elliptic curve with 160 bits $q$. By standard compression technique [27], the size of an element in group $G_{1}$ can be reduced to 65 bytes. The size of an element in $G_{2}$ is 1024 bits. So, the communication overhead of $\mathrm{SL}+\mathrm{BF}, \mathrm{LXJ}+\mathrm{BF}$ and our scheme are $3\left|G_{1}\right|+\left|\mathbb{Z}_{q}^{*}\right|+$ $|M A C|+2|m|$ bits $=3 * 65+20+20+|m| / 4$ bytes $=$ $235+|m| / 4$ bytes, $2\left|G_{1}\right|+\left|G_{2}\right|+2|m|$ bits $=2 * 65+$ $128+|m| / 4$ bytes $=258+|m| / 4$ bytes, and $\left|G_{1}\right|+\left|G_{2}\right|+$ $|m|$ bits $=65+128+|m| / 8$ bytes $=193+|m| / 8$ bytes, respectively. We can use the same method to compute the communication overhead at the 112-bit security level and 128-bit security level. We summarize the communication overhead at different security level in Fig. 3. Compared with $\mathrm{SL}+\mathrm{BF}$ and $\mathrm{LXJ}+\mathrm{BF}$, the communication overhead (here we assume that $|m|=1000$ bits) of our scheme is respectively reduced by $\frac{485-318}{485}=34.4 \%$ and $\frac{508-318}{508}=37.4 \%$ at the 80-bit security level. At the 112-bit security level, the communication overhead of our scheme is respectively reduced by $\frac{693-510}{693}=26.4 \%$ and $\frac{764-510}{764}=33.2 \%$. At the 128-bit security level, the communication overhead of our 

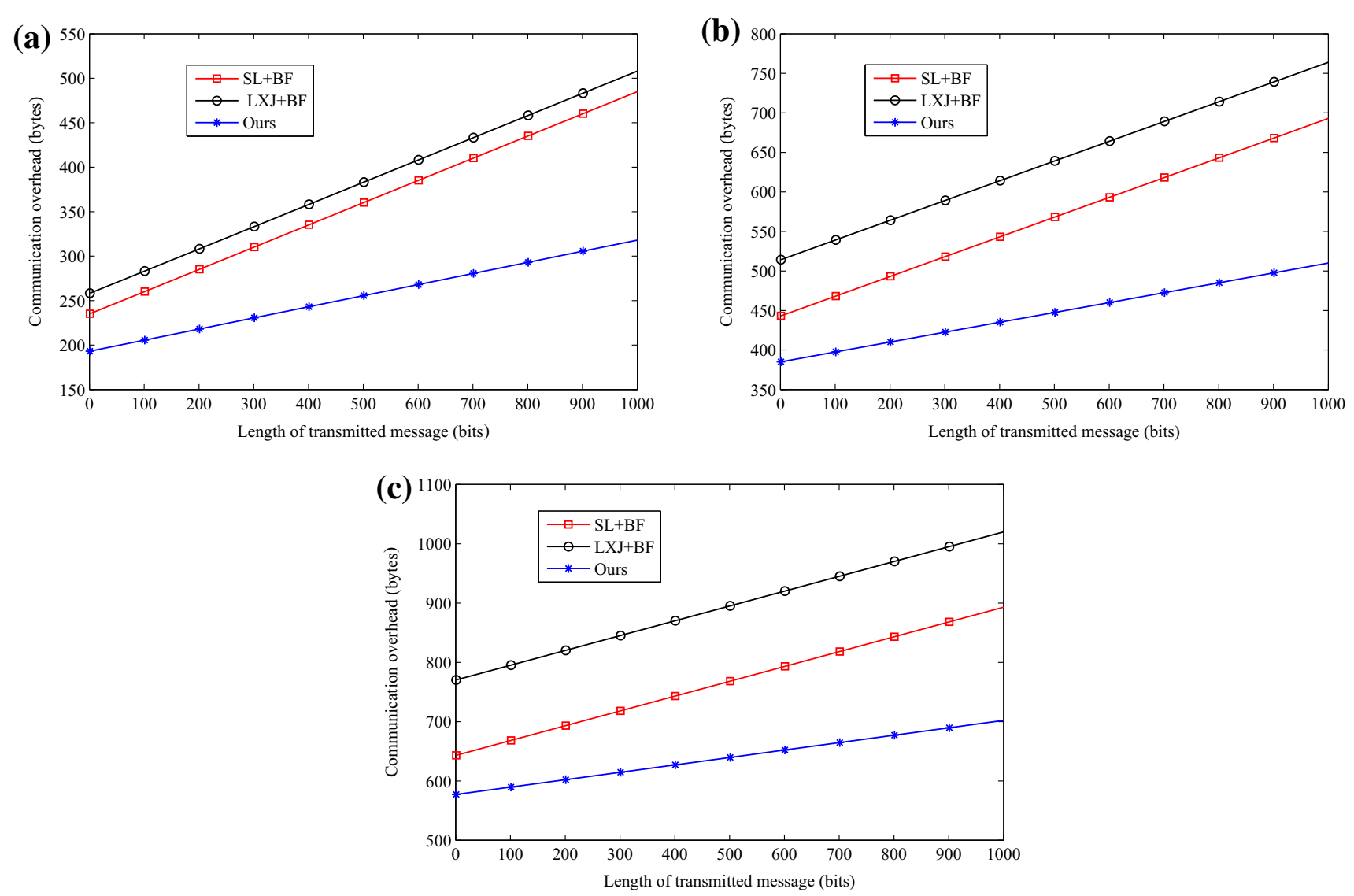

Fig. 3 The communication overhead versus length of transmitted message. a 80-bit security level. b 112-bit security level. c 128-bit security level

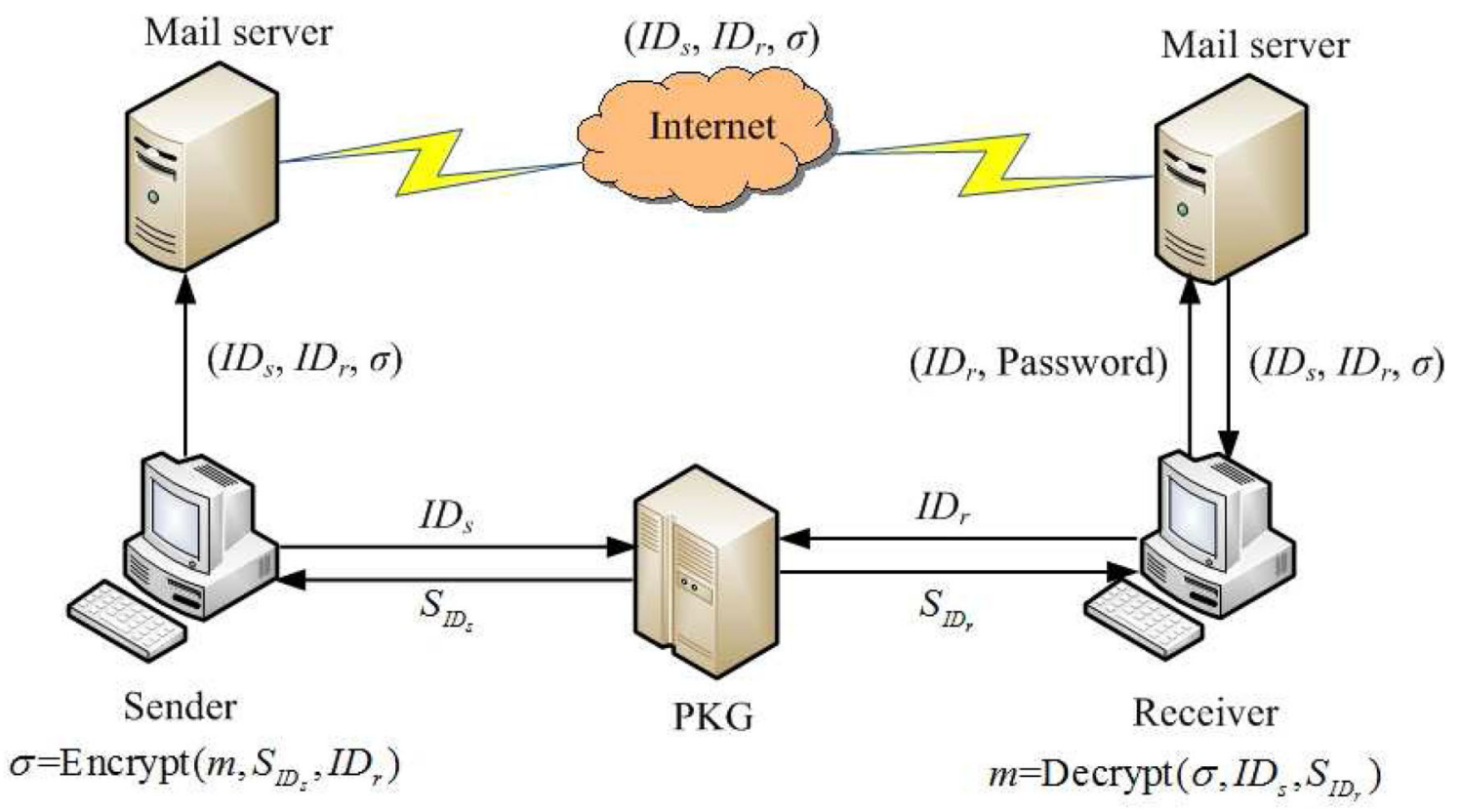

Fig. 4 A secure e-mail protocol 
scheme is respectively reduced by $\frac{893-702}{893}=21.3 \%$ and $\frac{1020-702}{1020}=31.1 \%$.

\section{Application}

In this section, we show an application of IBDEA to an e-mail system. A secure e-mail system should provides confidentiality and authentication. If we adopt "digital signature followed by encryption" method, the privacy of the sender may be infringed since the receiver can prove the source to any third party. The DAE solves this problem in a good way. Here we give a secure e-mail protocol using our IBDAE. This protocol consists of a sender, a receiver and mail servers and is described in Fig. 4. In this protocol, the identities of the sender and receiver are e-mail addresses. For example, the sender's identity $I D_{s}$ is alice@uestc. edu.cn and the receiver's identity $I D_{r}$ is bob@uestc.edu.cn. The sender first applies a private key $S_{I D_{s}}$ from the PKG and then runs Encrypt $\left(m, S_{I D_{s}}, I D_{r}\right)$ to get a ciphertext $\sigma$. The sender transmits its identity $I D_{s}$, the receiver's identity $I D_{r}$ and the ciphertext $\sigma$ to its mail server. Then the sender's mail server transfers $\left(I D_{s}, I D_{r}, \sigma\right)$ to the receiver's mail server. The receiver's mail server keeps $\left(I D_{s}, I D_{r}, \sigma\right)$ and waits for the receiver. When the receiver hopes to receive its mail, it submits its identity $I D_{r}$ and password to its mail server for authentication. If the receiver passes this authentication, the mail server sends the $\left(I D_{s}, I D_{r}, \sigma\right)$ to the receiver. The receiver can apply a private key $S_{I D_{r}}$ from the PKG and then runs Decrypt $\left(\sigma, I D_{s}, S_{I D_{r}}\right)$ to obtain the message $m$. Of course, the receiver can registers a private key $S_{I D_{s}}$ in advance. The hybrid technique is very suitable for sending a large e-mail.

\section{Conclusions}

In this paper, we proposed a hybrid IBDAE scheme that can achieve confidentiality and deniable authentication in a logical single step. Our construction is based on an IBDATK scheme and a DEM scheme. We proposed an IBDATK scheme using bilinear pairings and proved its security in the random oracle model. For typical security level, our scheme is at least 50.7 and $22.7 \%$ faster than two straightforward "deniable authentication followed by encryption" schemes, respectively. The communication overhead is respectively reduced at least 21.3 and $31.1 \%$. A secure e-mail protocol based on IBDAE was designed.

Acknowledgments This work is supported by the National Natural Science Foundation of China (Grant Nos. 61073176, 61272525, 61302161 and 61462048) and the Fundamental Research Funds for the Central Universities (Grant No. ZYGX2013J069).
Open Access This article is distributed under the terms of the Creative Commons Attribution 4.0 International License (http://creativecomm ons.org/licenses/by/4.0/), which permits unrestricted use, distribution, and reproduction in any medium, provided you give appropriate credit to the original author(s) and the source, provide a link to the Creative Commons license, and indicate if changes were made.

\section{Appendix}

\section{Data encapsulation mechanism (DEM)}

A DEM is a symmetric encryption scheme that consists of the following two algorithms.

- Enc is a deterministic encryption algorithm that takes as input a security parameter $k$, a key $K$ and a message $m \in\{0,1\}^{*}$, and outputs a ciphertext $c \in\{0,1\}^{*}$, where $K \in \mathcal{K}_{\text {DEM }}$ is a key in the given key space, and $m$ is a bit string of arbitrary length. We denote this as $c=$ $\operatorname{Enc}(K, m)$.

- Dec is a deterministic decryption algorithm that takes as input a key $K$ and a ciphertext $c$, and outputs the message $m \in\{0,1\}^{*}$ or a symbol $\perp$ to indicate that the ciphertext is invalid.

In this paper, we only require that a DEM is secure with respect to indistinguishability against passive attackers (IND-PA). Formally, this security notion is captured by the following game played between an adversary $\mathcal{A}$ and a challenger $\mathcal{C}$.

- Initial $\mathcal{A}$ runs on input $k$ and submits two equal length messages, $m_{0}$ and $m_{1}$.

- Challenge $\mathcal{C}$ selects a random key $K \in \mathcal{K}_{\text {DEM }}$ and a random bit $\beta \in\{0,1\}$, and sends $c=\operatorname{Enc}\left(K, m_{\beta}\right)$ to $\mathcal{A}$ as a challenge ciphertext.

- Guess $\mathcal{A}$ outputs a bit $\beta^{\prime}$ and wins the game if $\beta^{\prime}=\beta$.

The advantage of $\mathcal{A}$ is defined to be $\operatorname{Adv}(\mathcal{A})=\mid 2 \operatorname{Pr}\left[\beta^{\prime}=\right.$ $\beta]-1 \mid$, where $\operatorname{Pr}\left[\beta^{\prime}=\beta\right]$ is the probability that $\beta^{\prime}=\beta$.

Definition 7 An adversary $\mathcal{A}$ is said to be an $\left(\epsilon_{d e m}, t\right)$ attacker of a DEM scheme if $\mathcal{A}$ has advantage at least $\epsilon_{d e m}$ in the above game, runs in time at most $t$. A DEM scheme is said to be $\left(\epsilon_{d e m}, t\right)$-IND-PA secure if no $\left(\epsilon_{d e m}, t\right)$-attacker exists.

\section{Proof of Theorem 1}

Proof Our proof method is as follows. We define a sequence Game $_{0}$, Game $_{1}$, Game 2 of modified attack games. The only difference between games is how the environment responds to $\mathcal{A}$ 's oracle queries. 
Let $\sigma^{*}=\left(\psi^{*}, c^{*}\right)$ be the challenge ciphertext submitted to $\mathcal{A}$ by its challenge oracle that encrypts either $m_{0}$ or $m_{1}$ according to a bit $\beta$. Let $K^{*}$ denote the symmetric key used by the challenge oracle in the generation of the challenge ciphertext, or alternatively, the decapsulation of $\psi^{*}$ using the identities $I D_{s}$ and $I D_{r}$ that are chosen by the adversary. For any $i=0,1,2$, we let $S_{i}$ be the event that $\delta^{\prime}=\delta$ in game Game $_{i}$, where $\delta$ is the bit chosen by $\mathcal{A}$ 's challenge oracle and $\delta^{\prime}$ is the bit output by $\mathcal{A}$. This probability is taken over the random choices of $\mathcal{A}$ and those of $\mathcal{A}$ 's oracles.

We will use the following useful lemma from [28].

Lemma 1 ([28]) Let $E, E^{\prime}$, and $F$ be events defined on a probability space such that $\operatorname{Pr}[E \wedge \neg F]=\operatorname{Pr}\left[E^{\prime} \wedge \neg F\right]$. Then we have

$$
\left|\operatorname{Pr}[E]-\operatorname{Pr}\left[E^{\prime}\right]\right| \leq \operatorname{Pr}[F]
$$

Game $_{0}$ : We simulate the view of the adversary in a real attack by running the key extraction algorithm and using the resulting keys to respond to $\mathcal{A}$ 's queries. So the view of $\mathcal{A}$ is the same as it would be in a real attack. Therefore, we have

$\left|\operatorname{Pr}\left[S_{0}\right]-\frac{1}{2}\right|=\frac{1}{2} \epsilon_{\text {dae }}$.

Game 1 : In this game, we slightly modify how the decryption oracle responds to queries from $\mathcal{A}$. When a ciphertext $\sigma=(\psi, c)$, a sender' identity $I D_{i}$ and a receiver's identity $I D_{j}$ are presented to the decryption oracle after the invocation of the challenge encryption oracle, if $I D_{i}=I D_{s}$, $I D_{j}=I D_{r}$ and $\psi=\psi^{*}$, then the decryption oracle does not use the genuine decryption procedure for the hybrid scheme, instead it uses the key $K^{*}$ to decrypt $c$ and returns the result to the adversary $\mathcal{A}$.

Obviously this change has no impact on the adversary and so

$\operatorname{Pr}\left[S_{1}\right]=\operatorname{Pr}\left[S_{0}\right]$

Game$_{2}$ : In this game, we modify Game ${ }_{1}$ by replacing $K^{*}$ with a random key $K^{\prime}$ from $\mathcal{K}_{\text {DEM }}$. The result then follows from the following Lemmas 2 and 3.

Lemma 2 There exists a probabilistic polynomial-time algorithm $\mathcal{C}_{1}$, whose running time is essentially the same as that of $\mathcal{A}$, such that

$\left|\operatorname{Pr}\left[S_{2}\right]-\operatorname{Pr}\left[S_{1}\right]\right|=\epsilon_{\text {datk }}$.

Proof In this proof, we show how to construct an adversary $\mathcal{C}_{1}$ of the IBDATK to violate the assumed security against adaptive chosen ciphertext attack.
- Initial Given the system parameters param, $\mathcal{C}_{1}$ sends it to $\mathcal{A}$.

- Phase 1 When $\mathcal{A}$ makes a key extraction query on identity $I D, \mathcal{C}_{1}$ makes a key extraction query to its own key extraction oracle and forwards the answer to $\mathcal{A}$. When $\mathcal{A}$ makes an encryption query with a plaintext $m$, a sender's identity $I D_{i}$ and a receiver's identity $I D_{j}, \mathcal{C}_{1}$ follows the steps below.

1. Make a symmetric key generation query on $\left(I D_{i}, I D_{j}\right)$ to its own symmetric key generation oracle to obtain $K$.

2. Compute $c=\operatorname{DEM}$. Enc $(K, m)$.

3. Make a key encapsulation query on $c$ to its own key encapsulation oracle to obtain $\psi$.

4. Return the ciphertext $\sigma=(\psi, c)$ to $\mathcal{A}$.

When $\mathcal{A}$ makes a decryption query with a ciphertext $\sigma=$ $(\psi, c)$, a sender's identity $I D_{i}$ and a receiver's identity I $D_{j}, \mathcal{C}_{1}$ follows the steps below.

1. Make a key decapsulation query on $\left(\psi, c, I D_{i}, I D_{j}\right)$ to its own key decapsulation oracle to obtain $K$.

2. If $K=\perp$, return $\perp$ and stop.

3. Compute $m=\operatorname{DEM}$.Dec $(K, c)$ and return $m$.

- Challenge $\mathcal{A}$ generates two equal length plaintexts $m_{0}$ and $m_{1}$, a sender's identity $I D_{s}$ and a receiver's identity I $D_{r}$ on which it wishes to be challenged. $\mathcal{C}_{1}$ follows the steps below.

1. Submit $I D_{s}$ and $I D_{r}$ to its challenger to obtain $K_{\beta}$, where $\beta \in\{0,1\}$.

2. Pick a random bit $\delta$ from $\{0,1\}$.

3. Compute $c^{*}=\mathrm{DEM}$.Enc $\left(K_{\beta}, m_{\delta}\right)$.

4. Submit $c^{*}$ to its challenger to obtain $\psi^{*}$.

5. Return the ciphertext $\sigma^{*}=\left(\psi^{*}, c^{*}\right)$ to $\mathcal{A}$.

- Phase $2 \mathcal{A}$ can ask a polynomially bounded number of queries adaptively again as in phase 1 with the restriction that it cannot make a key extraction query on $I D_{r}$ and it cannot make a decryption query on $\sigma^{*}=\left(\psi^{*}, c^{*}\right)$ to obtain the corresponding plaintext.

- Guess $\mathcal{A}$ outputs $\delta^{\prime}$. If $\delta^{\prime}=\delta, \mathcal{C}_{1}$ outputs $\beta^{\prime}=1$ indicating $K_{\beta}$ is the real key; otherwise it outputs $\beta^{\prime}=0$ indicating $K_{\beta}$ is a random key.

When $K_{\beta}$ is the real key, $\mathcal{A}$ is run exactly as it would be run in Game 1 . This means that

$\operatorname{Pr}\left[S_{1}\right]=\operatorname{Pr}\left[\delta^{\prime}=\delta \mid \beta=1\right]=\operatorname{Pr}\left[\beta^{\prime}=1 \mid \beta=1\right]$

When $K_{\beta}$ is the random key, $\mathcal{A}$ is run exactly as it would be in Game2. This means that

$\operatorname{Pr}\left[S_{2}\right]=\operatorname{Pr}\left[\delta^{\prime}=\delta \mid \beta=0\right]=\operatorname{Pr}\left[\beta^{\prime}=1 \mid \beta=0\right]$. 
From the definition of security for IBDATK, we have

$$
\begin{aligned}
\epsilon_{\text {datk }}= & \left|2 \operatorname{Pr}\left[\beta^{\prime}=\beta\right]-1\right|=\mid \operatorname{Pr}\left[\beta^{\prime}=1 \mid \beta=1\right] \\
& -\operatorname{Pr}\left[\beta^{\prime}=1 \mid \beta=0\right] \mid .
\end{aligned}
$$

So the result holds.

Lemma 3 There exists a probabilistic polynomial-time algorithm $\mathcal{C}_{2}$, whose running time is essentially the same as that of $\mathcal{A}$, such that

$\left|\operatorname{Pr}\left[S_{2}\right]-\frac{1}{2}\right|=\frac{1}{2} \epsilon_{\text {dem }}$.

Proof To construct such a $\mathcal{C}_{2}$ we simply run $\mathcal{A}$ as it would be run in game Game2. We run the key extraction step so we can respond to $\mathcal{A}$ 's queries before it calls its challenge encryption oracle. When $\mathcal{A}$ calls its challenge encryption oracle with two messages $m_{0}$ and $m_{1}$, a sender's identity $I D_{s}$ and a receiver's identity $I D_{r}$, we simply relay $m_{0}$ and $m_{1}$ to the challenge encryption oracle of $\mathcal{C}_{2}$ to obtain $c^{*}$. We then make a symmetric key generation query and a key encapsulation query to obtain $K^{*}$ and $\psi^{*}$, respectively. We discard $K^{*}$ and return $\sigma^{*}=\left(\psi^{*}, c^{*}\right)$ to $\mathcal{A}$.

In this simulation $\mathcal{A}$ is run by $\mathcal{C}_{2}$ in exactly the same manner as the former would be run in game Game 2 ; moreover, $\operatorname{Pr}\left[S_{2}\right]$ corresponds exactly to the probability that $\mathcal{C}_{2}$ correctly determines the hidden bit of its challenge encryption oracle since $\mathcal{C}_{2}$ outputs whatever $\mathcal{A}$ outputs. The result follows.

\section{Proof of Theorem 2}

Proof Suppose that $\mathcal{F}$ is an adversary who can breaks the DA-CMA security of the IBDAE scheme with advantage $\epsilon_{d a e}$. We can use this to construct another algorithm $\mathcal{C}$ that can break the DA-CMA security of the IBDATK with advantage at least $\epsilon_{\text {datk }}$ too. $\mathcal{C}$ runs as follow.

- Initial Given the system parameters param, $\mathcal{C}$ sends it to $\mathcal{F}$.

- Attack When $\mathcal{F}$ makes a key extraction query on identity $I D, \mathcal{C}$ makes a key extraction query to its own key extraction oracle and forwards the answer to $\mathcal{F}$. When $\mathcal{F}$ makes an encryption query with a plaintext $m$, a sender's identity $I D_{i}$ and a receiver's identity $I D_{j}, \mathcal{C}$ follows the steps below.

1. Make a symmetric key generation query on $\left(I D_{i}, I D_{j}\right)$ to its own symmetric key generation oracle to obtain $K$.

2. Compute $c=\mathrm{DEM}$. $\operatorname{Enc}(K, m)$.

3. Make a key encapsulation query on $c$ to its own key encapsulation oracle to obtain $\psi$.

4. Return the ciphertext $\sigma=(\psi, c)$ to $\mathcal{F}$.
When $\mathcal{F}$ makes a decryption query with a ciphertext $\sigma=$ $(\psi, c)$, a sender's identity $I D_{i}$ and a receiver's identity $I D_{j}, \mathcal{C}$ follows the steps below.

1. Make a key decapsulation query on $\left(\psi, c, I D_{i}, I D_{j}\right)$ to its own key decapsulation oracle to obtain $K$.

2. If $K=\perp$, return $\perp$ and stop.

3. Compute $m=\mathrm{DEM} \cdot \operatorname{Dec}(K, c)$ and return $m$.

- Forgery $\mathcal{F}$ outputs $\left(\sigma^{*}, I D_{s}, I D_{r}\right)$, where $\sigma^{*}=$ $\left(\psi^{*}, c^{*}\right) . \mathcal{C}$ outputs $\left(\psi^{*}, \tau^{*}, I D_{s}, I D_{r}\right)$, where $\tau^{*}=c^{*}$.

Obviously, this simulation is perfect. If $\mathcal{F}$ wins the DACMA game for the IBDAE, $\mathcal{C}$ have the same probability to win the DA-CMA game for the IBDATK.

\section{Proof of Theorem 3}

Proof $\mathcal{C}$ receives a random instance $(P, a P, b P, c P, \theta)$ of the DBDH problem and attempts to decide whether $\theta=$ $\hat{e}(P, P)^{a b c}$ or not. $\mathcal{C}$ will run $\mathcal{A}$ as a subroutine and play $\mathcal{A}$ 's challenger in Definition 3. $\mathcal{A}$ will consult $\mathcal{C}$ for answers to the random oracles $H_{1}, H_{2}$ and $H_{3}$. Roughly speaking, these answers are randomly generated, but to maintain the consistency and to avoid collision. $\mathcal{C}$ keeps three lists $L_{1}$, $L_{2}$ and $L_{3}$ respectively to store the answers. The following assumptions are made.

1. $\mathcal{A}$ will ask for $H_{1}(I D)$ before $I D$ is used in any key extraction queries, symmetric key generation queries, key encapsulation queries and key decapsulation queries.

2. An encapsulation returned from a key encapsulation query will not be used by $\mathcal{A}$ in a key decapsulation query.

3. The sender's identity and the receiver's identity are different in Encap and Decap algorithms. This assumption is called irreflexivity assumption in [11]. Such an assumption makes our proof simple.

- Initial $\mathcal{C}$ gives $\mathcal{A}$ the system parameters with $P_{\text {pub }}=$ $c P$. Note that $c$ is unknown to $\mathcal{C}$. This value simulates the master secret key for the PKG.

- Phase $1 \mathcal{A}$ performs a polynomially bounded number of queries.

- $H_{1}$ queries $\mathcal{C}$ first selects a number $\lambda \in\left\{1,2, \ldots, q_{H_{1}}\right\}$ randomly. $\mathcal{A}$ asks a polynomially bounded number of $H_{1}$ queries on identities of its choice. At the $\lambda$-th $H_{1}$ query, $\mathcal{C}$ answers by $H_{1}\left(I D_{\lambda}\right)=b P$. For queries $H_{1}\left(I D_{i}\right)$ with $i \neq \lambda, \mathcal{C}$ selects $d_{i} \in \mathbb{Z}_{q}^{*}$ randomly, inserts the pair $\left(I D_{i}, d_{i}\right)$ in the list $L_{1}$ and answers $H_{1}\left(I D_{i}\right)=d_{i} P$.

- $\mathrm{H}_{2}$ and $\mathrm{H}_{3}$ queries When $\mathcal{A}$ asks queries on these hash values, $\mathcal{C}$ checks the corresponding list. If an entry for the query is found, the same answer will be 
given to $\mathcal{A}$; otherwise, a randomly chosen value will be used as the answer to $\mathcal{A}$. The query and answer will then be inserted in the list.

- Key extraction queries $\mathcal{A}$ submits an identity $I D_{i}$ to $\mathcal{C}$. If $I D_{i}=I D_{\lambda}$, then $\mathcal{C}$ fails and stops. If $I D_{i} \neq I D_{\lambda}$, then the list $L_{1}$ must contain a pair $\left(I D_{i}, d_{i}\right)$ for some $d_{i}$ (this indicates $\mathcal{C}$ previously answered $\left.H_{1}\left(I D_{i}\right)=d_{i} P\right)$. The private key for $I D_{i}$ is $d_{i} P_{\text {pub }}=d_{i} c P$. It is computed by $\mathcal{C}$ and returned to $\mathcal{A}$.

- Symmetric key generation queries $\mathcal{A}$ produces a sender's identity $I D_{i}$ and a receiver's identity $I D_{j}$. If $I D_{i} \neq I D_{\lambda} . \mathcal{C}$ first obtains the private key $S_{I D_{i}}$ by running a key extraction query. Then $\mathcal{C}$ answers the query by running $(K, \omega)=\operatorname{Sym}\left(S_{I D_{i}}, I D_{j}\right)$ algorithm. Note that $\mathcal{C}$ needs to store $\omega$ and to overwrite any previous value. If $I D_{i}=I D_{\lambda}$ and hence I $D_{j} \neq I D_{\lambda}$ by the irreflexivity assumption, $\mathcal{C}$ first chooses $x$ from $\mathbb{Z}_{q}^{*}$ randomly and computes $z=$ $\hat{e}\left(P_{p u b}, Q_{I D_{j}}\right)^{x}$. Then $\mathcal{C}$ runs the $H_{2}$ simulation algorithm to find $K=H_{2}(z)$. Finally, $\mathcal{C}$ gives $K$ to $\mathcal{A}$. Note that the $\omega$ is $\left(x, z, I D_{i}, I D_{j}\right)$ in this case.

- Key encapsulation queries $\mathcal{A}$ produces an arbitrary tag $\tau$. $\mathcal{C}$ checks whether there exists a stored value $\omega$. If not, it returns $\perp$ and stops. Otherwise we have the following two cases to consider. The first case is $I D_{i} \neq I D_{\lambda}$. In this case, $\mathcal{C}$ just runs $\psi=$ $\operatorname{Encap}(\omega, \tau)$ and returns the result to $\mathcal{A}$. The second case is $I D_{i}=I D_{\lambda}$ and hence $I D_{j} \neq I D_{\lambda}$ by the irreflexivity assumption. In this case, $\mathcal{C}$ first runs the $H_{3}$ simulation algorithm to get $u=H_{3}(\tau, z)$ and then computes $R=u Q_{I D_{i}}$ and $T=z \cdot \hat{e}\left(R, S_{I D_{j}}\right)(\mathcal{C}$ could obtain $S_{I D_{j}}$ from the key extraction algorithm because $\left.I D_{j} \neq I D_{\lambda}\right)$. Finally, $\mathcal{C}$ gives $\psi=(R, T)$ to $\mathcal{A}$.

- Key decapsulation queries $\mathcal{A}$ selects an encapsulation $\psi$, a tag $\tau$, a sender's identity $I D_{i}$ and a receiver's identity $I D_{j}$. If $I D_{j}=I D_{\lambda}, \mathcal{C}$ always answers $\mathcal{A}$ that $(\psi, \tau)$ is invalid. If $\mathcal{A}$ previously asked the hash value $H_{3}(\tau, z)$, where $z=T / \hat{e}\left(R, S_{I D_{j}}\right)$, and $\mathcal{C}$ answered $u$ that satisfies $R=u Q_{I D_{i}}, \mathcal{C}$ will fail. In this instance, $(\psi, \tau)$ is actually valid from $\mathcal{A}$ 's point of view. The probability of this instance is at most $1 / 2^{k}$. If $I D_{j} \neq I D_{\lambda}, \mathcal{C}$ first obtains $S_{I D_{j}}$ from the key extraction algorithm and then sends the result of $\operatorname{Decap}\left(\psi, \tau, I D_{i}, S_{I D_{j}}\right)$ to $\mathcal{A}$.

- Challenge $\mathcal{A}$ decides when phase 1 ends. $\mathcal{A}$ produces a sender's identity $I D_{s}$ and a receiver's identity $I D_{r}$ on which it hopes to be challenged. If $\mathcal{A}$ has asked a key extraction query on $I D_{\lambda}, \mathcal{C}$ fails. On the other hand, if $\mathcal{A}$ does not choose $I D_{r}=I D_{\lambda}$ as target identity, $\mathcal{C}$ fails too. $\mathcal{C}$ first chooses $T^{*} \in G_{2}$, sets $R^{*}=a P$ and computes
$z^{*}=T^{*} / \theta$ (where $\theta$ is $\mathcal{C}$ 's candidate for the DBDH problem). Then $\mathcal{C}$ runs the $\mathrm{H}_{2}$ simulation algorithm to find $K_{1}=H_{2}\left(z^{*}\right) . \mathcal{C}$ chooses $K_{0} \in \mathcal{K}_{\text {IBDATK }}$ and a bit $\beta \in\{0,1\}$ randomly, and sends $K_{\beta}$ to $\mathcal{A}$. $\mathcal{A}$ then sends a tag $\tau^{*}$ to $\mathcal{C}$. Finally, $\mathcal{C}$ gives the encapsulation $\psi^{*}=\left(R^{*}, T^{*}\right)$ to $\mathcal{A}$.

- Phase $2 \mathcal{A}$ can ask a polynomially bounded number of queries adaptively again as in phase 1 with the restriction that it cannot make a key extraction query on $I D_{r}$ and cannot make a decapsulation query on $\left(\psi^{*}, \tau^{*}\right)$ to obtain the corresponding key.

- Guess $\mathcal{A}$ outputs a bit $\beta^{\prime}$ for which it believes the relation $\left(K_{\beta}, \omega^{*}\right)=\operatorname{Sym}\left(S_{I D_{s}}, I D_{r}\right)$ and $\psi^{*}=\operatorname{Encap}\left(\omega^{*}, \tau^{*}\right)$ hold. At this moment, if $\beta^{\prime}=\beta, \mathcal{C}$ returns 1 that denotes $\theta=\hat{e}(P, P)^{a b c}$; otherwise, it returns 0 that denotes $\theta \neq$ $\hat{e}(P, P)^{a b c}$.

We now assess $\mathcal{C}$ 's probability of success. Note that $\mathcal{C}$ only fails in providing a consistent simulation because one of the following independent events:

$E_{1} \mathcal{A}$ does not choose $I D_{\lambda}$ as the receiver's identity in the challenge phase.

$E_{2} \mathcal{A}$ has made a key extraction query on $I D_{\lambda}$.

$E_{3} \mathcal{C}$ aborts in a key decapsulation query because of rejecting an valid encapsulation.

We know that $\operatorname{Pr}\left[\neg E_{1}\right]=1 / q_{H_{1}}$ and $\operatorname{Pr}\left[E_{3}\right] \leq q_{d} / 2^{k}$. In addition, we know that $\neg E_{1}$ implies $\neg E_{2}$.

Since

$$
\begin{aligned}
p_{1} & =\operatorname{Pr}\left[\beta^{\prime}=\beta \mid\left(K_{\beta}, \omega^{*}\right)=\operatorname{Sym}\left(S_{I D_{s}}, I D_{r}\right) \text { and } \psi^{*}\right. \\
& \left.=\operatorname{Encap}\left(\omega^{*}, \tau^{*}\right)\right] \\
& =\frac{\epsilon+1}{2}-\frac{q_{d}}{2^{k}}
\end{aligned}
$$

and

$p_{0}=\operatorname{Pr}\left[\beta^{\prime}=i \mid \theta \in_{R} G_{2}\right]=\frac{1}{2}$ for $i=0,1$,

We have

$$
\begin{aligned}
\operatorname{Adv}(\mathcal{C}) & =\frac{\left|p_{1}-p_{0}\right|}{q_{H_{1}}}=\left(\frac{\epsilon+1}{2}-\frac{q_{d}}{2^{k}}-\frac{1}{2}\right)\left(\frac{1}{q_{H_{1}}}\right) \\
& =\frac{\epsilon-q_{d} / 2^{k-1}}{2 q_{H_{1}}}
\end{aligned}
$$

The bound on $\mathcal{C}$ 's computation time comes from the fact that $\mathcal{C}$ needs $O\left(q_{s}+q_{e}+q_{d}\right)$ pairing operations in the symmetric key generation queries, key encapsulation queries and key decapsulation queries. 


\section{Proof of Theorem 4}

Proof Here we use the forking lemma [29] to prove the security of our scheme. To use the forking lemma, we need to show how our scheme fits into the signature scheme described in [29], the simulation step in which the signature can be simulated without the sender's private key (and thus, also without the master secret key), and how we can solve BDH problem based on the forgery.

First, we observe that our scheme satisfies the requirement described in [29]. During the encapsulation of a tag $\tau$, the tuple $\left(\sigma_{1}, u, \sigma_{2}\right)$ is generated that corresponds to the required three-phase honest-verifier zero-knowledge identification protocol, where $\sigma_{1}=z$ is the commitment of the prover, $u=H_{3}(\tau, z)$ is the hash value depending on $\tau$ and $\sigma_{1}$ substituted for the verifier's challenge, and $\sigma_{2}=T$ is the response of the prover that relies on $\sigma_{1}, u$ and the sender's private key $S_{I D_{s}}$.

Next, we need to show a simulation step that supplies a faithful simulation to the forger $\mathcal{F}$ and how to solve the BDH problem by interacting with $\mathcal{F}$. $\mathcal{C}$ receives a random instance $(P, a P, b P, c P)$ of the $\mathrm{BDH}$ problem and is required to compute $h=\hat{e}(P, P)^{a b c}$. $\mathcal{C}$ will run $\mathcal{F}$ as a subroutine and plays $\mathcal{F}$ 's challenger in the game of Definition 4 . We describe this process as follows.

- Initial $\mathcal{C}$ runs Setup algorithm with a security parameter $k$ and sends the system parameters with $P_{p u b}=c P$ to $\mathcal{F}$.

- Attack $\mathcal{C}$ answers $\mathcal{F}$ 's queries according to the method in Theorem 3 except that the simulator should choose two different random numbers $\chi, \xi \in\left\{1,2, \ldots, q_{H_{1}}\right\}$ in advance. At the $\chi$-th $H_{1}$ query, $\mathcal{C}$ answers by $H_{1}\left(I D_{\chi}\right)=$ $a P$. At the $\xi$-th $H_{1}$ query, $\mathcal{C}$ answers by $H_{1}\left(I D_{\xi}\right)=b P$. If we let $I D_{\lambda}=\left\{I D_{\chi}, I D_{\xi}\right\}$, the simulation process is the same as that in Theorem 3.

- Forgery $\mathcal{F}$ outputs a quaternion $\left(\psi^{*}, \tau^{*}, I D_{\chi}, I D_{\xi}\right)$, where $\psi^{*}=\left(R^{*}, T^{*}\right)$. We coalesce the identities $I D_{\lambda}=$ $\left\{I D_{\chi}, I D_{\xi}\right\}$ and the tag $\tau^{*}$ into a "generalized" forged $\operatorname{tag}\left(I D_{\lambda}, \tau^{*}\right)$ so as to hide the identity-based aspect of the DA-CMA attack, and simulate the setting of an identityless adaptive-CMA existential forgery for which the forking lemma is proven. From the the forking lemma [29], if $\mathcal{F}$ is a sufficiently efficient forger in the above interaction, then we can construct a Las Vegas machine $\mathcal{F}^{\prime}$ that outputs two signed tags $\left(\left(I D_{\lambda}, \tau^{*}\right), u^{*}, \psi^{*}\right)$ and $\left(\left(I D_{\lambda}, \tau^{*}\right), \bar{u}^{*}, \bar{\psi}^{*}\right)$ with $u^{*} \neq \bar{u}^{*}$ and the same commitment $z^{*}$. To solve the BDH problem based on the machine $\mathcal{F}^{\prime}$ derived from $\mathcal{F}$, we construct a machine $\mathcal{C}^{\prime}$ as follows.

1. $\mathcal{C}^{\prime}$ runs $\mathcal{F}^{\prime}$ to get two distinct signatures $\left(\left(I D_{\lambda}, \tau^{*}\right)\right.$, $\left.u^{*}, \psi^{*}\right)$ and $\left(\left(I D_{\lambda}, \tau^{*}\right), \bar{u}^{*}, \bar{\psi}^{*}\right)$.
2. $\mathcal{C}^{\prime}$ computes $\hat{e}(P, P)^{a b c}$ as $\left(T^{*} / \bar{T}^{*}\right)^{1 /\left(u^{*}-\bar{u}^{*}\right)}$.

From the forking lemma [29] and the lemma on the relationship between given-identity attack and chosen-identity attack [8], if $\mathcal{F}$ succeeds in time $t$ with probability $\epsilon \geq$ $5\left(q_{e}+1\right)\left(q_{e}+q_{H_{3}}\right) q_{H_{1}} /\left(2^{k}-1\right)$, then $\mathcal{C}^{\prime}$ can solve the $\mathrm{BDH}$ problem in expected time $t \leq 60343 q_{H_{3}} q_{H_{1}} 2^{k} t / \epsilon\left(2^{k}-1\right)$. Note that the coefficient is changed because the simulator should choose two different identities in advance.

\section{References}

1. Maimut, D., \& Reyhanitabar, R. (2014). Authenticated encryption: Toward next-generation algorithms. IEEE Security \& Privacy, 12(2), 70-72.

2. Wu, T. S., \& Lin, H. Y. (2014). Provably secure proxy convertible authenticated encryption scheme based on RSA. Information Sciences, 278, 577-587.

3. Xie, Q. (2012). Provably secure convertible multi-authenticated encryption scheme. IET Information Security, 6(2), 65-70.

4. Shamir, A. (1984). Identity-based cryptosystems and signature schemes. In Proc CRYPTO'84, LNCS (Vol. 196, pp. 47-53). Springer.

5. Boneh, D., \& Franklin, M. (2001). Identity-based encryption from the weil pairing. In Proc CRYPTO 2001, LNCS (Vol. 2139, pp. 213-229). Springer.

6. Park, J. H., \& Lee, D. H. (2015). An efficient IBE scheme with tight security reduction in the random oracle model. Designs, Codes and Cryptography. doi:10.1007/s10623-015-0035-0.

7. Hess, F. (2003). Efficient identity based signature schemes based on pairings. In Proc SAC 2002, LNCS (Vol. 2595, pp. 310-324). Springer.

8. Cha, J. C., \& Cheon, J. H. (2003). An identity-based signature from gap Diffie-Hellman groups. In Proc PKC 2003, LNCS (Vol. 2567, pp. 18-30). Springer.

9. Hsu, C. L., \& Lin, H. Y. (2011). New identity-based key-insulated convertible multi-authenticated encryption scheme. Journal of Network and Computer Applications, 34(5), 1724-1731.

10. Lin, H. Y., \& Hsu, C. L. (2011). A novel identity-based keyinsulated convertible authenticated encryption scheme. International Journal of Foundations of Computer Science, 22(3), 739756.

11. Boyen, X. (2003). Multipurpose identity-based signcryption: A swiss army knife for identity-based cryptography. In Proc CRYPTO 2003, LNCS (Vol. 2729, pp. 383-399). Springer.

12. Barreto, P. S. L. M., Libert, B., McCullagh, N., \& Quisquater, J. J. (2005). Efficient and provably-secure identity-based signatures and signcryption from bilinear maps. In Proc ASIACRYPT 2005, LNCS (Vol. 3788, pp. 515-532). Springer.

13. Lin, C., Tang, F., Ke, P., Harn, L., \& Zhang, S. (2014). Secure universal designated verifier identity-based signcryption. Security and Communication Networks, 7(2), 434-444.

14. Cramer, R., \& Shoup, V. (2003). Design and analysis of practical public-key encryption schemes secure against adaptive chosen ciphertext attack. SIAM Journal on Computing, 33(1), 167-226.

15. Abe, M., Gennaro, R., \& Kurosawa, K. (2008). Tag-KEM/DEM: A new framework for hybrid encryption. Journal of Cryptology, 21(1), 97-130.

16. Manulis, M., Poettering, B., \& Stebila, D. (2014). Plaintext awareness in identity-based key encapsulation. International Journal of Information Security, 13(1), 25-49. 
17. Kang, L., Tang, X. H., \& Liu, J. F. (2014). Tight chosen ciphertext attack (CCA)-secure hybrid encryption scheme with full public verifiability. SCIENCE CHINA Information Sciences, 57(11), 114.

18. Bentahar, K., Farshim, P., Malone-Lee, J., \& Smart, N. P. (2008). Generic constructions of identity-based and certificateless KEMs. Journal of Cryptology, 21(2), 178-199.

19. Abdalla, M., Catalano, D., \& Fiore, D. (2014). Verifiable random functions: Relations to identity-based key encapsulation and new constructions. Journal of Cryptology, 27(3), 544-593.

20. Raimondo, M. D., \& Gennaro, R. (2009). New approaches for deniable authentication. Journal of Cryptology, 22(4), 572-615.

21. Li, F., \& Takagi, T. (2013). Cryptanalysis and improvement of robust deniable authentication protocol. Wireless Personal Communications, 69(4), 1391-1398.

22. Shi, Y., \& Li, J. (2005). Identity-based deniable authentication protocol. Electronics Letters, 41(5), 241-242.

23. Kar, J. (2013). ID-based deniable authentication protocol based on Diffie-Hellman problem on elliptic curve. International Journal of Network Security, 15(5), 357-364.

24. Li, F., Xiong, P., \& Jin, C. (2014). Identity-based deniable authentication for ad hoc networks. Computing, 96(9), 843-853.

25. PBC Library. http://crypto.stanford.edu/pbc/.

26. Daemen, J., \& Rijmen, V. (2002). The design of Rijndael: AESThe Advanced Encryption Standard. Berlin: Springer.

27. Shim, K. A. (2012). CPAS: An efficient conditional privacypreserving authentication scheme for vehicular sensor networks. IEEE Transactions on Vehicular Technology, 61(4), 1874-1883.

28. Shoup, V. (2001). OAEP reconsidered. In Proc CRYPTO 2001, LNCS (Vol. 2139, pp. 239-259). Springer.

29. Pointcheval, D., \& Stern, J. (2000). Security arguments for digital signatures and blind signatures. Journal of Cryptology, 13(3), 361396

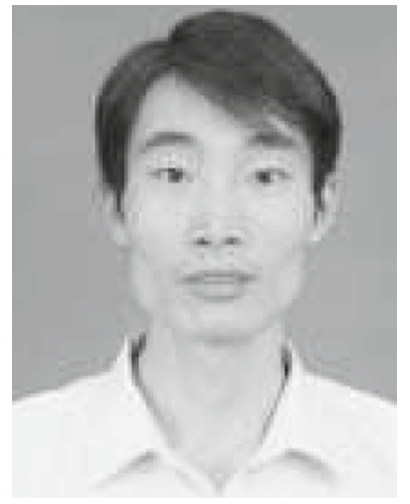

Fagen $\mathbf{L i}$ is now an associate professor in the School of Computer Science and Engineering, University of Electronic Science and Technology of China, Chengdu, P.R. China. He received his Ph.D. degree in Cryptography from Xidian University, Xi'an, P.R. China in 2007. From 2008 to 2009, he was a postdoctoral fellow in Future University-Hakodate, Hokkaido, Japan, which is supported by the Japan Society for the Promotion of Science. He worked as a research fellow in the Institute of Mathematics for Industry, Kyushu University, Fukuoka, Japan from 2010 to 2012. His recent research interests include cryptography and network security. He has published more than 70 papers in the international journals and conferences. He is a member of the IEEE.

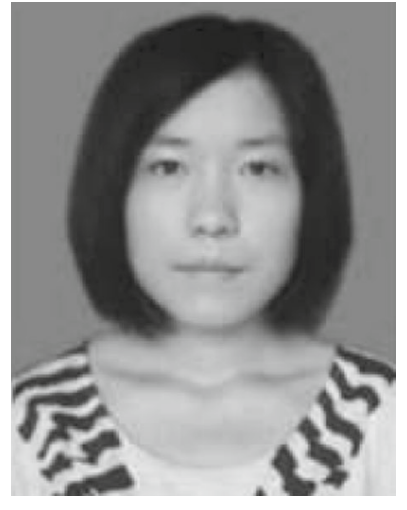

Zhaohui Zheng received her B.S. degree in Computer Science from Henan Normal University, Zhengzhou, P.R. China in 2012. She is now a master student in the School of Computer Science and Engineering, University of Electronic Science and Technology of China, Chengdu, P.R. China. Her research interests include cryptography and information security.

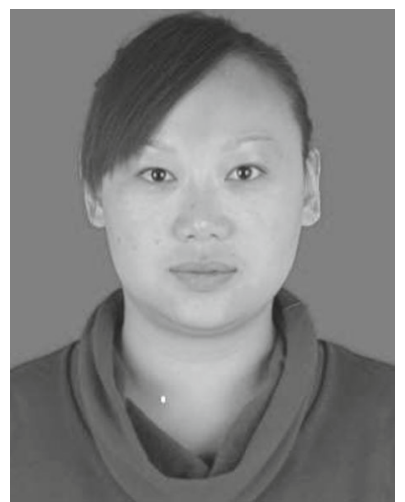

Chunhua Jin is now a Ph.D. student in the School of Computer Science and Engineering, University of Electronic Science and Technology of China, Chengdu, P.R. China. Her research interests include cryptography and network security. 